\title{
La neurociencia en la primera infancia
}

\author{
Neuroscience in early childhood
}

\author{
Claudia Natalia Bodero Cáceres ${ }^{1,2}$ \\ ${ }^{1}$ Colegio Champagnat, Lima, ${ }^{2}$ Centro de Capacitación Integral CBC, Lima
}

\section{RESUMEN}

La educación en el Perú y la formación de los niños, en especial en la primera infancia, viene siendo actualmente criticada tanto por la formación familiar, la preparación de maestros como por la calidad de la enseñanza. El 2013 trajo para nuestro sistema educativo un resultado desalentador como el de la prueba PISA (Programa Internacional para la Evaluación de Estudiantes), que analizó el rendimiento de los alumnos de 15 años de edad en matemáticas, lenguaje y ciencias, y ubicó al Perú en uno de los últimos puestos entre 65 países. Este artículo analiza la importancia de la formación del niño desde que la madre se encuentra en gestación hasta cuando el menor acude a formar parte del sistema educativo. Pone énfasis en la formación de valores, emocionales, sociales, etc., de manera que el niño potencie su capacidad de ser feliz y sea buen ciudadano. Es importante que un educador entienda a las neurociencias como una forma amplia de conocer al cerebro -cómo es, cómo aprende, cómo procesa, registra, conserva y evoca una información, entre otros aspectos- para mejorar las propuestas y experiencias de aprendizaje que se dan en el aula. Los docentes tienen la posibilidad de ayudar a desarrollar el potencial de todos y cada uno de nuestros alumnos dado que es, en la etapa escolar, cuando más moldeable y plástico se encuentra el cerebro. Es vital aprovechar ese momento y la oportunidad que se nos ofrece. Podemos hacer mucho desde la escuela. El futuro de la sociedad reside en la mente humana y el tipo de conexiones que establecemos interna y externamente.

Palabras clave: Neurociencia, educación, primera infancia, familia, niño, valores.

\section{ABSTRACT}

Education in Perú and the education of children, especially in early childhood, is currently being criticized both by family formation, teacher preparation and the quality of education. 2013 brought a discouraging result for our educational system, such as the PISA (Programa Internacional para la Evaluacion de Estudiantes), which analyzed the performance of 15 years old students in mathematics, language and science, and placed Perú in one of the last places among 65 countries. This article analyzes the importance of the child's education from the moment the mother is pregnant until the child comes to be part of the education system. Emphasizes the formation of values, emotional, social, etc., so that the child enhances their ability to be happy and be a good citizen. It is important that an educator understands neurosciences as a broad way of knowing the brain -how it is, how it learns, how it processes, records, preserves and evokes information, among other aspects- to improve the proposals and learning experiences that occur in the classroom. The teachers have the possibility of helping to develop the potential of each and every one of our students since it is, in the school stage, when the brain is more moldable and plastic. It is vital to take advantage of that moment and the opportunity that is offered to us. We can do a lot from school. The future of society lies in the human mind and the type of connections we establish internally and externally.

Keywords: Neuroscience, education, early childhood, family, child, values.

Historial del artículo:

Recibido, 15 de febrero de 2016; aceptado, 10 de junio de 2017; disponible en línea, 25 de junio de 2017

* Licenciada en Educación Inicial, docente del Colegio Champagnat, investigadora del Centro de Capacitación Integral CBC

Correo: cbodero11@gmail.com 


\section{INTRODUCCIÓN}

La primera infancia es la etapa que abarca desde el nacimiento hasta los ocho años de edad, y es la más significativa en la formación del individuo porque en ella se estructuran las bases del desarrollo humano y la personalidad, sobre las que se consolidarán y perfeccionarán las sucesivas etapas. Es en esta etapa donde las estructuras neurofisiológicas y psicológicas están en pleno proceso de maduración, en este sentido la calidad y cantidad de influencias que reciban los niños y niñas de su entorno familiar, socioeconómico y cultural las moldearán de una forma casi definitiva. De ahí que la educación en esta etapa llega a ejercer una acción determinante porque precisamente actúa sobre estructuras que están en plena fase de maduración y desarrollo.

La etapa está marcada por un notable crecimiento físico y significativo desarrollo sensorial y perceptivo. Es determinante en el despertar de habilidades emocionales, intelectuales y sociales, así como en el desarrollo del lenguaje y de las más diversas formas de expresión como cantar, bailar, moverse, llorar, pintar y explorar, que son muy significativas en esta etapa de la vida del ser humano.

Diversas investigaciones han señalado que las experiencias físicas, sociales, emocionales, cognitivas y sensoriales, a las que están expuestos los niños y niñas, son críticas y cruciales para la organización neurológica de su sistema nervioso y para el desarrollo de su cerebro, porque dejan huellas para toda la vida.

El artículo explica este proceso del desarrollo del niño en la primera infancia, a partir de una visión académica y desde la experiencia como educadora.

\section{Inicio de la primera infancia}

La primera infancia va desde el nacimiento hasta los 8 años de edad, comprende el nacimiento y el primer año de vida, los años preescolares y en la transición hasta la escolarización. Esta amplia y valiosa etapa está marcada por un notable crecimiento físico y significativo desarrollo sensorial y perceptivo.

Los educadores somos los gestores de los cambios o de la continuidad respecto a la forma en que actuamos en el aula y debemos ser capaces de poder trasformar nuestras prácticas en un espacio de creación e innovación o quienes afiancemos las tradicionales maneras de abordar la tarea educativa.

Hoy los cambios sociales y aportes de la neurociencia obligan al docente a ser protagonista de su formación y a brindar diversas formas de enseñar y también de aprender.
Un aspecto importante será desarrollar la capacidad de sistematizar los aspectos más importantes del proceso de aprendizaje realizado, a fin de mejorar la socialización y trasmitir a las familias y comunidad educativa las propuestas y logros del aprendizaje alcanzados con los niños y niñas.

La neuroeducación es uno de los factores que ayudará a mejorar la calidad de nuestros sistemas educativos y promoverá mejores programas de la primera infancia, pero para ello necesitamos repensar el perfil del educador de modo que sepa orientar la transformación.

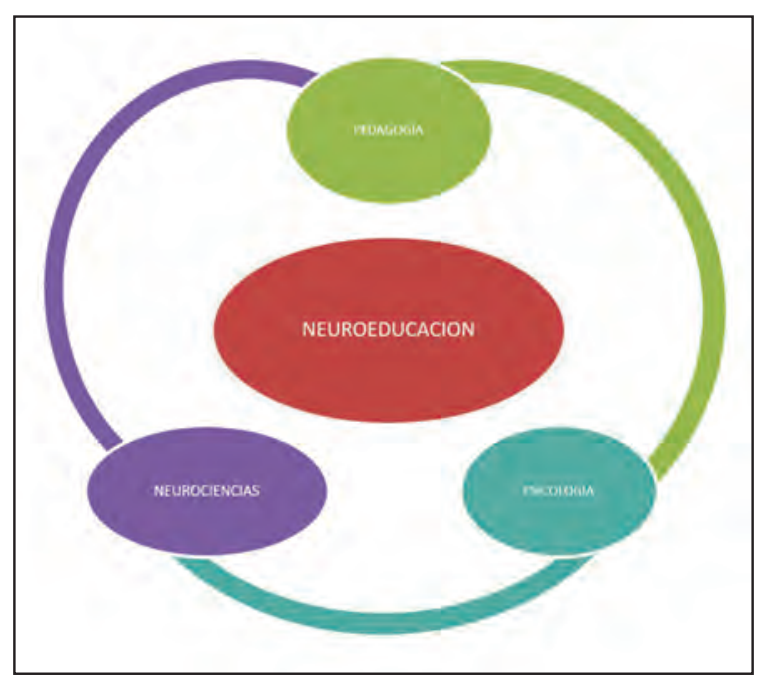

Figura 1. La neuroeducación.

El primer factor que hace la diferencia en la primera infancia está relacionado directamente con la formación inicial y continúa con el apoyo de los padres y educadores, el rol de ellos es vital para el crecimiento y desarrollo del cerebro. Existen diversos estudios que explican esta relación en los primeros años de vida.

Entre 0 y 8 años la posibilidad de modificar estructural y funcionalmente el cerebro, por su uso o desuso, debe hacer que los adultos consideren muy importante el tipo de educación que se le brinde al niño en esta etapa del desarrollo. La neuroeducación nos ayudará a educar teniendo el cerebro en mente

Todas las acciones y muchas otras habilidades más son los resultados de una serie de cambios que van permitiendo segundo tras segundo que el cerebro se vuelva más funcional.

- El refinamiento del sistema de conexión entre las neuronas.

- La poda de aquellas que no tienen función para la red.

- La formación de nuevas conexiones. 
- La ramificación dendrítica y del proceso de mielinización.

Las decisiones que se tomen en relación con la atención y educación durante esta etapa de la vida pueden tener una consecuencia duradera, marcando el ritmo y la calidad, no solo del desarrollo de un individuo sino también del contexto social donde está inmerso. Los padres, educadores, psicólogos, asistentes sociales y toda persona que conviva con un niño o niña, lleva consigo una enorme responsabilidad: conocer cómo es el proceso de desarrollo en la infancia, para saber cómo atenderlo y estimularlo (Campos, 2010a). La atención integral y la educación de calidad en la primera infancia -sea en un contexto familiar, comunitario o institucional, formal o no formal- tiene un significativo impacto en la salud mental y física de los niños y niñas, en el desarrollo de múltiples habilidades, en la disminución de los índices de mortalidad materno infantil, de anemia, de la repitencia y deserción de los centros educativos antes de finalizar la primaria, conllevando también a la disminución de las brechas ocasionadas por la pobreza en las poblaciones más vulnerables.

Las investigaciones relacionadas indican que a pesar de todos los argumentos fundamentados, los gobiernos recién empiezan a prestar la debida atención a la primera infancia. Un porcentaje muy alto de países carecen de programas de educación y atención integral para los niños y niñas menores de tres años, evidenciando que estos programas no constituyen una prioridad para una cantidad significativa de gobiernos.

\section{¿Qué debemos conocer de la construcción del cerebro en la etapa prenatal?}

- Hablar de la primera infancia es hablar paralelamente de los mecanismos de desarrollo cerebral que subyacen en este proceso y que empiezan tres semanas después de la concepción en el vientre materno.

- El desarrollo del sistema nervioso y del cerebro obedecen a una programación genética que tiene unos principios básicos de organización, lo que nos permite tener conocimiento de la secuencia fabulosa de los sucesos que ocurren durante el desarrollo cerebral del feto.

- En resumen, todas las regiones del cerebro en la etapa prenatal y las funciones que desempeña, se fortalece a partir de las conexiones que se van estableciendo entre las células que las componen.

- A través de conexiones, las neuronas empiezan a comunicarse, fenómeno conocido como sinaptogénesis. La transferencia de la información entre neuronas sucede en sitios especializados llamados sinapsis, que puede ser de tipo eléctrico o químico.

- La mielinización de las fibras nerviosas, luego de establecidas las sinapsis, es altamente relevante para el seguimiento y fortalecimiento de las funciones. A mayor mielinización, mayor será la funcionalidad de los circuitos neuronales.

- Aunque los genes controlan el proceso de mielinización, los factores ambientales pueden afectar su grado y calidad. La desnutrición, tanto de la madre gestante como del niño, es uno de los factores que afecta al proceso de mielinización, ya que las células gliales también son sensibles a la calidad de la nutrición.

\section{Los primeros meses de vida son momentos decisivos para el cerebro}

- Al nacer, el cerebro de un bebe pesa aproximadamente la cuarta parte de lo que llegará a pesar cuando sea un adulto, y esto gracias a todo lo que ha sucedido dentro del vientre materno.

- Un nacimiento bajo condiciones apropiadas juega un papel decisivo, permite al bebe una adaptación armónica con el nuevo ambiente que está lleno de sonidos, luces, olores, temperaturas y texturas; y le facilita la tarea de encontrar a un "cerebro externo" que lo adapte al nuevo mundo.

- Durante la infancia temprana, los circuitos de la certeza cerebral poseen un estado de alta plasticidad (adaptaciones de los circuitos neuronales frente al aprendizaje o a las contingencias) que facilita su modificación.

- El sueño es considerado un agente importante para el desarrollo del cerebro, ya que supone el financiamiento de aprendizajes de carácter bioquímico. La consolidación de la memoria de largo plazo se realiza cuando el cerebro pasa por el sueño profundo (REM) y ello dado desde la primera infancia.

- Otro factor de suma importancia a tener en cuenta en el desarrollo y maduración cerebral del infante es la nutrición. Indiscutiblemente, el alimento más completo y rico en todos los aspectos para los bebes es la leche materna. Investigaciones realizadas han demostrado que los ácidos grasos, encontrados en la leche materna, son ideales para el desarrollo del sistema nervioso, además de poseer cualidad inmunitaria y psicológica.

- El rol del afecto en esta etapa temprana, además del sueño y la nutrición, es fundamental para la maduración neurobiológica y el desarrollo emocional, motriz y cognitivo de los niños.

- A los pocos meses después del nacimiento, los padres podrán observar varias de las habilidades, fruto de un cerebro cada día más desarrollado. Teniendo en cuenta que las experiencias van a influenciar en el cableado cerebral, es de suma importancia propiciar oportunidades, recursos y ambientes adecuados. 
- Diversos estudios concluyeron que en los primeros años de vida, el proceso de conexión sináptica y la plasticidad cerebral son exuberantes, pues, a diferencia del cuerpo, el cerebro no añade tantas células después del nacimiento pero sí hace crecer las prolongaciones de las mismas, provocando un sistema de comunicaciones fenomenal.

- Al segundo año el niño habrá reunido innumerables experiencias sensoriales y motoras, las cuales habrán ayudado a su cerebro a alcanzar el siguiente nivel de su potencial: la habilidad de representar objetos, personas o eventos a través de símbolos mentales.

\section{De 3 a 8 años edad}

En estos años se produce una maduración significativa del sistema nervioso, muscular y óseo, por lo que los factores como la desnutrición, la privación de afectos y de estímulos sensoriales tienen una enorme incidencia en los procesos de crecimiento y desarrollo no solo del cuerpo, sino también del cerebro.

- El cerebro realiza 1,8 millones de nuevas sinapsis por segundo entre los dos meses de gestación y los dos años de edad, y que el $83 \%$ del crecimiento dendrítico ocurre después del nacimiento (Campos, 2010b).

- El resultado de la relación afectiva madre-niño fundamentalmente va a tener una relevancia importante en la configuración de la personalidad de los niños y niñas y en sus habilidades sociales, que le permitan reconocerse a sí mismo, comenzando por la propia imagen, y descubrir al final de esta etapa la existencia de los otros y así asumir una conducta de grupo.

- Entre los 2 y 5 años, el programa genético pone en marcha un proceso gradual de poda sináptica regional que alcanza su pico entre los cinco y seis años de edad, en esta primera etapa del ciclo vital. Esta poda sináptica sigue el principio de eficiencia: se eliminan las conexiones funcionales inútiles, inactivas y no aptas para el desafío cognitivo y social que va a venir.

- Entre los 6 y 7 años, nuevas y solidas sinapsis remplazan a las que fueron eliminadas, más eficientes en sus funciones. Este proceso incluye lo verbal como lo musical. Por ello es importante estimularlos con lecturas de cuentos, conversaciones y música.

- Cuanto más un educador comprenda la relación entre la maduración de los diferentes circuitos neurales y el surgimiento o fortalecimiento de las capacidades, habilidades y destrezas, de mayor calidad será la planificación de sus actividades, las propuestas de aprendizaje e inclusive, el instrumento y contenido de la evaluación de los aprendizajes y del desarrollo infantil.

Mora (2013) autor de Neuroeducación manifiesta que solo se puede aprender aquello que se ama, uno de los primeros manuales dedicados a este tema y todo un fenómeno de ventas, manifiesta que conocer los códigos de funcionamiento del cerebro ha permitido demostrar, por ejemplo, la importancia de la curiosidad y la emoción para adquirir conocimientos; que el deporte es esencial para fijar el aprendizaje y también que en el cerebro hay "ventanas de conocimiento" que se abren y se cierran de acuerdo con las etapas de la vida.

Kandel (1997), uno de los más reconocidos neurocientíficos en la actualidad, afirma que si bien el cerebro está apto para aprender y estructurar el lenguaje, sea hablado o escrito y en cualquier idioma, hay un período sensible para la adquisición de este aprendizaje, que corresponde entre los dos y ocho años aproximadamente, marcado por la fuerte relación con el entorno y las personas que lo componen. Kandel afirma también que a partir de esta etapa, la capacidad de adquisición del lenguaje se reduce fuertemente.

\section{Las dimensiones del desarrollo humano}

El desarrollo del ser humano empieza en el vientre materno y es el fruto de una relación armoniosa entre genética y entorno; es un proceso dinámico, continuo y que dura toda la vida, tiene la peculiar característica de ser acumulativo $y$, en este sentido, la primera infancia viene a ser la base que sustentará todas las estructuras posteriores. También se caracteriza por ser multidimensional, lo que nos lleva a comprender la existencia de varios campos de acción que hay que incidir en ellos.

Diferentes investigadores establecen en consenso que se puede definir claramente seis dimensiones básicas del desarrollo humano:

En la primera infancia se produce el gran despegue de aprendizaje de habilidades que conforman cada dimensión, y el cerebro el responsable de su funcionalidad.

Utilizar el cuerpo, el movimiento, explorar con todos los sentidos, emocionarse, descubrir, imitar, son algunas de las herramientas que utilizan los niños y niñas por indicación de estos sistemas neuronales de aprendizaje de un cerebro esforzándose por estructurarse y aprender.

Es muy importante que los padres, educadores y toda la sociedad tengan conocimiento de los elementos que desarrollan, potencian o estacan el proceso de estas dimensiones para que puedan actuar de forma adecuada y ser agentes de promoción del desarrollo infantil. 


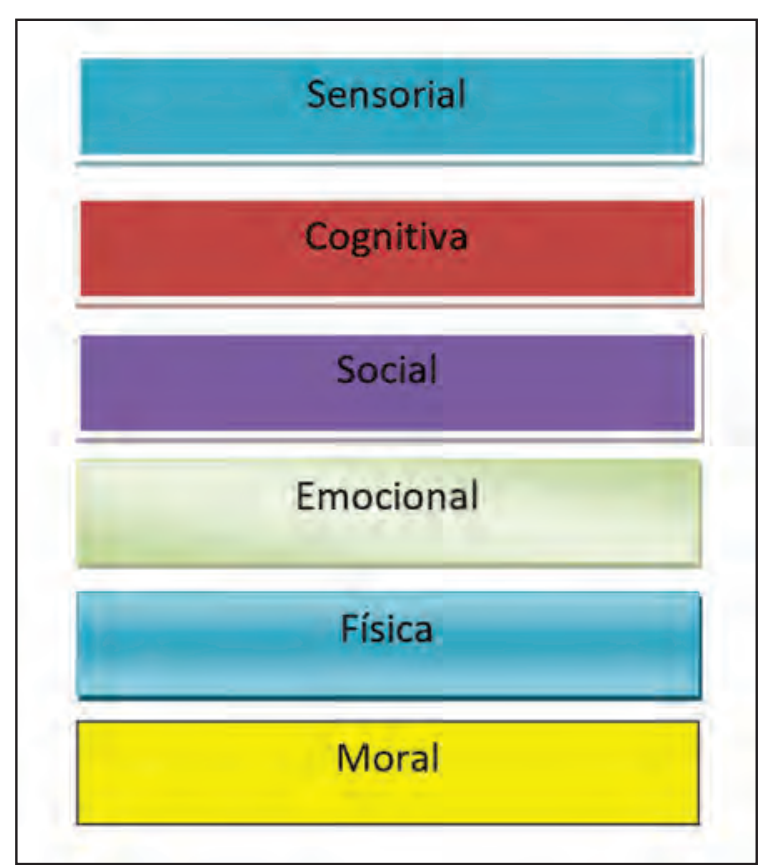

Figura 2. Dimensiones básicas del desarrollo humano (elaboración propia).

\section{CONCLUSIONES}

- La primera infancia es la base del desarrollo humano: incidir en esta etapa aumenta significativamente las probabilidades de mejorar nuestros sistemas educativos.

- Todas habilidades y capacidades son frutos de un cerebro en constante aprendizaje y desarrollo, $y$, a medida que el conocimiento relacionado al funcionamiento del cerebro humano vaya siendo más accesible a los educadores, el proceso de aprendizaje se volverá más efectivo y significativo tanto para un educador como para el alumno.

- Las neurociencias, más que una propuesta, debe ser una ciencia que aporte nuevos conocimientos al educador, así como lo hace la psicología, por ejemplo, con el propósito de proveerle de suficiente fundamento para innovar y transformar su práctica pedagógica.

- Es muy importante que un educador entienda a las neurociencias como una forma amplia de conocer al cerebro -cómo es, cómo aprende, cómo procesa, registra, conserva y evoca una información, entre otros aspectos- para que así pueda mejorar las propuestas y experiencias de aprendizaje que se dan en el aula.

- Los docentes tienen la posibilidad de ayudar a desarrollar el potencial de todos los alumnos dado que es, en la etapa escolar, cuando más moldeable y plástico se encuentra el cerebro. Es vital aprovechar ese momento que se presenta. Es posible hacer mucho desde la escuela. El futuro de la sociedad reside en la mente humana y el tipo de conexiones que se establece interna y externamente.

\section{REFERENCIAS BIBLIOGRÁFICAS}

Campos, A. L. (2010). Primera infancia: una mirada desde la neuroeducación. Lima: Cerebrum \& OEA.

Campos, A. L. (2010). Neuroeducación: cómo educar para que el cerebro aprenda. Lima: Cerebrum Ediciones.

Kandel , E., Jessell , T., \& Schwartz , J. (2005). Neurociencia y conducta. Madrid: Pearson Prentice Hall.

Mora, F. (2013). Neuroeducación, solo se puede aprender aquello que se ama. Madrid: Alianza Editorial. 